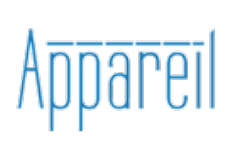

Appareil

9| 2012

Penser l'art, penser l'histoire

\title{
Idolâtrie, idéologie, iconologie
}

Vers une archéologie des Visual Studies

\section{Ralph Dekoninck}

\section{(2) OpenEdition}

Journals

Édition électronique

URL : http://journals.openedition.org/appareil/1458

DOI : 10.4000/appareil. 1458

ISSN : 2101-0714

Éditeur

MSH Paris Nord

Référence électronique

Ralph Dekoninck, « Idolâtrie, idéologie, iconologie », Appareil [En ligne], 9 | 2012, mis en ligne le 02 juillet 2012, consulté le 30 juillet 2020. URL : http://journals.openedition.org/appareil/1458 ; DOI : https:// doi.org/10.4000/appareil.1458

Ce document a été généré automatiquement le 30 juillet 2020.

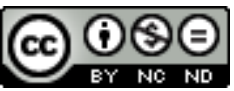

Appareil est mis à disposition selon les termes de la Licence Creative Commons Attribution - Pas d'Utilisation Commerciale - Pas de Modification 4.0 International. 


\title{
Idolâtrie, idéologie, iconologie
}

\author{
Vers une archéologie des Visual Studies
}

\author{
Ralph Dekoninck
}

\section{NOTE DE L'AUTEUR}

Cet article a fait l'objet d'une traduction anglaise, voir "Idolatry, Ideology, Iconology: Towards an Archaeology of the Visual Studies", in B. Baert, A.-S. Lehmann et J. Van den Akkerveken (ed.), New Perspectives in Iconology, Visual Studies and Anthropology, Leuven, ASP, 2012, p. 17-22.

1 On ne peut être que frappé par la quasi absence dans le champ des Visual Studies de réflexions approfondies sur les relations que cette discipline ou ce courant de recherche entretient avec l'iconologie, tradition par rapport à laquelle on serait en droit de s'attendre qu'elle se positionne ${ }^{1}$. Pour ne mentionner qu'un seul exemple qui me paraît représentatif de ce silence, on peut citer la synthèse historiographique de Margaret Dikovitskaya ${ }^{2}$ : l'index qui clôt le volume n'offre aucune entrée pour le mot « iconologie ». Il y a là de quoi interpeller tout historien de l'art s'intéressant aux Visual Studies. Et si l'on approfondit quelque peu l'enquête, cette première impression ne fait que se confirmer. Certes, il faut bien reconnaître qu'il y a quelques exceptions : qu'il suffise de citer les travaux de Michael Ann Holly, grande spécialiste de Panofsky³, mais qui fut également l'une des premières promotrices, avec Norman Bryson et Keith Moxey, d'un tournant critique en histoire de l'art orienté vers la visual theory, ce qui peut être traduit par «théorie du visuel» et/ou par "théorie visuelle ${ }^{4}$ ». Mais là encore, on reste, après ces lectures, quelque peu sur sa faim, le rapport de l'iconologie aux Visual Studies étant, à mon sens, insuffisamment problématisé ou à tout le moins insuffisamment mis en perspective.

Comment donc interpréter ce quasi silence? Faut-il y voir une forme de meurtre du père ? Sans s'engager dans une psychanalyse épistémologique, il apparaît évident que les Visual Studies ont cherché à prendre leurs distances vis-à-vis du prétendu logocentrisme de l'iconologie s'appuyant sur un certain paradigme linguistique. De ce 
point de vue, et la chose est suffisamment connue, le pictorial ou iconic turn est bien une réponse au linguistic turn. Mais une telle explication est bien entendu trop simpliste et occulte l'inspiration puisée par les Visual Studies chez les premiers maîtres à penser de l'histoire de l'art, le pictorial turn n'étant à vrai dire qu'un pictorial return, retour, aux yeux de certains, à une forme d'âge d'or incarné par des figures comme Aby Warburg.

On peut bien sûr renverser la perspective en se demandant si l'inverse est également vrai : l'iconologie telle qu'elle se pratique aujourd'hui se laisse-t-elle imprégner des idées véhiculées par les Visual Studies? Je ne retiendrai ici qu'un seul exemple, celui de l'ouvrage publié à l'occasion du centenaire de la naissance d'Erwin Panofsky: Meaning in the Visual Arts: Views from the Outside, ouvrage paru en 1995, soit à un moment d'affirmation des Visual Studies aux États-Unis ${ }^{5}$. Ces points de vue "extérieurs" qu'évoque le titre désignent à vrai dire les grands domaines de recherche avec lesquels l'iconologie n'a cessé d'entretenir des liens étroits : anthropologie, histoire, littérature, science, musique et film. On a là une répartition assez classique des savoirs et des arts que l'émergence et l'affirmation des Visual Studies à cette même époque viennent complètement bouleverser, s'inscrivant en cela dans la mouvance lancée par les artistes eux-mêmes dès le début du $\mathrm{xx}^{\mathrm{e}}$ siècle. Certes, dans la rubrique littérature, autre trait assez symptomatique, on compte un article de W. J. T. Mitchell intitulé "What is Visual Culture ?"6 texte qui peut apparaître programmatique puisqu'il y pose les jalons du programme qui sera le sien par la suite et qui va largement inspirer le mainstream des Visual Studies. On ne trouve toutefois dans cet article aucune tentative d'articulation entre les Visual Studies et l'iconologie.

Et pourtant c'est bien à Mitchell que l'on doit l'un des tous premiers essais de réactualisation de l'iconologie. C'est en effet l'un des premiers à avoir procédé à une archéologie des Visual Studies et, plus précisément, à avoir affronté la question des relations généalogiques entre cette discipline émergeante et l'iconologie. Il l'a fait dès 1986 dans un ouvrage intitulé Iconology: Image, Text, Ideology ${ }^{7}$. Si, de l'aveu même de Mitchell, cette iconologie fait aujourd'hui figure pour lui d'archéologie, et si bon nombre de ses idées ont évolué depuis, on ne peut toutefois s'empêcher d'y repérer les germes de bien des développements ultérieurs.

Reprenant la conception panofskienne de l'iconologie comme interprétation de l'horizon symbolique de l'image, son but est «de généraliser les ambitions interprétatives de l'iconologie en lui demandant de considérer l'idée d'image ellemême ${ }^{8}$ ». Ce qui revient à renouer avec le sens étymologique du mot "iconologie »: l'iconologie est l'étude du logos des images. Cette dernière formule est à comprendre dans un double sens: premièrement, comme l'étude "de ce qu'il y a à dire des images », soit la tradition des « écrits sur l'art » qui remonte aux Images de Philostrate, tradition principalement centrée sur la description et l'interprétation des arts visuels; deuxièmement, comme l'étude de ce que disent les images, à savoir leur façon de persuader, de narrer et de décrire, comme si elles étaient douées de parole. ${ }^{9}$ Cette double perspective résume parfaitement les deux dimensions qui resteront constitutives, voire coextensives des Visual Studies : l'attention portée à la fois sur ce qui est dit des images et sur ce que les images nous disent. Or il apparaît qu'au moment où les Visual Studies voient le jour dans les années 1980, c'est la première dimension qui compte aux yeux de Mitchell : celle du discours porté sur les images, alors que c'est plutôt la seconde dimension qui l'emporte dans ses plus récents travaux ${ }^{10}$. L'ouvrage de 1986 est en effet consacré en grande partie aux diverses idéologies qui ont pu sous- 
tendre les différentes pensées de l'image. De ce point de vue, l'iconologie s'avère être non pas tant une science de l'image qu'une "psychologie politique des images ${ }^{11}$ " s'interrogeant sur les valeurs qui leur sont attribuées, depuis la haine jusqu'à l'adoration. De fait, l'idée d'image est dépendante de systèmes de pouvoirs et de valeurs dont l'étude fut et reste l'un des objectifs majeurs des Visual Studies.

6 L'un des exemples retenus par Mitchell, et que je souhaiterais développer à mon tour, est celui des théories de l'idolâtrie, à savoir l'une des idéologies les plus persistantes touchant les images de l'«autre ». Étonnamment, l'un des premiers à dénoncer la nature idéologique de l'idolâtrie n'est autre que Luther, qui rejeta une définition ontologique de l'idole comme fausse image pour reconnaître derrière cette notion non pas une réalité en soi mais une catégorie mentale projetée sur la réalité :

les images ne sont ni bonnes, ni mauvaises, [...] on doit laisser aux gens la liberté d'en avoir ou de n'en pas avoir [...], car nous sommes obligés de reconnaître [...] que l'on peut trouver des gens qui font des images un bon usage. L'abus ne se trouve pas en elle-même, mais dans celui qui les utilise ${ }^{12}$.

7 En détournant la fameuse formule de Duchamp appliquée à l'art, on pourrait ainsi dire que le regardeur fait l'idole.

8 Cette idée selon laquelle l'idolâtrie est avant tout une question de projection et d'introjection sera reprise et développée par Francis Bacon sur l'œuvre duquel se penche Mitchell. Les «idoles de l'esprit» (mentis idola) désignent pour le philosophe anglais toutes les fausses idées et notions (notiones falsae), qui sont à vrai dire de fausses représentations ${ }^{13}$. Il n'est donc plus question de condamner une image qui serait fausse ou dangereuse par nature, mais la manière dont l'entendement humain, comme un faux miroir recevant les rayons de manière irrégulière, déforme la nature des choses en les mélangeant avec sa propre nature ${ }^{14}$. Mitchell écrit à ce propos : «Le culte des idoles dans les sombres bosquets et les cavernes de la superstition païenne font place aux croyances superstitieuses dans le pouvoir des idoles mentales qui résident dans l'obscure caverne de notre crâne ${ }^{15}$ ». De ce point de vue, l'idolâtrie peut bien être identifiée à une forme d'idéologie, au sens où l'on entend ce terme aujourd'hui. De façon plus générale, la religion, dans sa dimension superstitieuse, fut perçue, à partir $\mathrm{du} \mathrm{XVIII}^{\mathrm{e}}$ siècle, comme un système de fausses représentations, dont l'origine est à trouver dans l'imagination, cette « folle du logis » pour reprendre la célèbre formule de Malebranche.

9 Ce qui conduit Mitchell à conclure que le concept d'idéologie est enraciné dans le concept d'imagerie ${ }^{16}$ ou, plus précisément, qu'il est fondé, comme le terme lui-même le suggère, sur la notion d'entités mentales ou d'idées qui fournissent ses matériaux à la pensée.

Si ces idées sont comprises comme des images - c'est-à-dire comme des signes graphiques ou picturaux imprimés ou projetés sur le support de la conscience alors l'idéologie, comme science des idées, est réellement une iconologie, une théorie de l'imagerie ${ }^{17}$.

10 Avant de renvoyer à la structure des valeurs et des intérêts qui informent toute représentation de la réalité, l'idéologie était originellement la science de ces représentations. D'une certaine manière, une fausse connaissance des vraies images, ce qu'était l'idolâtrie, est remplacée par une vraie connaissance des fausses images, ce qu'est l'idéologie, laquelle n'est autre dès lors qu'une forme d'iconologie, idée et image se confondant jusqu'à un certain point. 
11 Cependant, si l'on veut comparer jusqu'au bout idéologie et iconologie, il convient de noter - et sur ce point je quitte les réflexions de Mitchell pour m'engager sur le terrain de recherche qui est le mien - que le déplacement dans la signification du mot « idéologie », depuis l'étude elle-même jusqu'au sujet de cette étude, est inverse à celui opéré pour le mot "iconologie" qui est caractérisé par la même ambiguïté. Si l'on remonte en effet au sens premier du mot, dont l'invention peut être attribuée à Cesare Ripa, on peut constater qu'il désigne à l'origine non pas encore une science portant sur les images (« what we say about images »), mais un savoir produit par les images ellesmêmes («what images say »), ou plus exactement un savoir qui se présentait et était alors conçu comme un art. En d'autres termes, l'art des images précède la science des images, ou pour le dire dans les termes de l'époque : l'ars imaginum est antérieur à la scientia imaginum.

Cette dernière expression mérite de retenir l'attention. Elle est en fait utilisée, pour la première fois à ma connaissance, par un jésuite français, Nicolas Caussin, dans un ouvrage intitulé Electorum symbolorum $(1618)^{18}$, ouvrage consacré à la théorie du symbole et qui procède à ce qu'on pourrait nommer une rhétoricisation de l'image symbolique $^{19}$. On s'éloigne ainsi d'une conception néoplatonicienne valorisant une connaissance intuitive de la vérité voilée-révélée par l'image ${ }^{20}$, pour se rapprocher d'une conception logique et rhétorique ${ }^{21}$. S'il y a theoria du symbole, ce n'est donc plus au sens étymologique d'une contemplation de la vérité qui viendrait s'incarner dans les signes, mais au sens d'une construction d'un savoir, non pas encore de l'image ou sur l'image, mais bien par l'image. Ce savoir par l'image, Ripa lui donne le nom d'Iconologia, terme désignant les images et les figures inventées par l'esprit humain non plus tant pour voiler que pour révéler la vérité. C'est l'art « de donner un corps à nos pensées, et d'ainsi les rendre visibles ${ }^{22}$ ». Qui plus est, pour lui comme pour beaucoup d'autres humanistes avant lui, cette conception correspond à une volonté de sauver les images antiques de la condamnation qui pèse encore sur elles, surtout en cette fin $\mathrm{du} \mathrm{XVI}^{\mathrm{e}}$ siècle, dans le contexte de la Contre-Réforme. Ainsi Ripa reconnaît-il que ces images furent souvent utilisées par les Anciens, qui inventèrent de nombreuses figures pour leurs dieux. Or ces figures, il les interprète comme « les vêtements couvrant les parties de la philosophie traitant de la procréation et putréfaction des choses naturelles, de la forme des cieux et de l'influence des étoiles, de la solidité de la terre et d'autres choses semblables ${ }^{23}$ ". Tous les dieux païens sont ainsi convertis en symboles d'idées abstraites, tandis que les fables antiques sont approchées comme des philosophies déguisées. On pourrait ainsi dire que, du domaine de la superstition religieuse, on glisse vers le royaume de la raison, laquelle est certes encore enchantée par l'imagination. L'image comme objet d'une fausse croyance devient la meilleure façon de révéler la vérité. Celle-ci n'est cependant plus immanente au monde, mais apparaît désormais comme une construction de la raison ou, à tout le moins, la manière la plus efficace de penser et de communiquer cette pensée. On pourrait dire en quelque sorte que l'iconologie triomphe sur l'idolâtrie.

13 Mais elle ne triomphe pas pour autant sur l'idéologie, comprise cette fois en son sens actuel, étant donné que le savoir produit par les images est conçu non pas tant pour instruire que pour convaincre, emporter l'adhésion et pousser à l'action. Cette dimension pragmatique de l'image agissante est fondamentalement idéologique car étroitement liée à la question du pouvoir dans son rapport au savoir. Si l'on veut maintenant observer un déplacement de cette pratique de l'image agissante vers une 
théorie de l'image pensante, d'une iconopraxis à une iconologie, il faut s'engager plus avant dans le XvII ${ }^{\mathrm{e}}$ siècle. Un exemple qui me semble assez représentatif est celui de la Philosophie des images du jésuite français Claude-François Ménestrier ${ }^{24}$. Ce titre est celui que ce savant iconologue donne à sa vaste entreprise d'inventaire et de systématisation de tous les langages figurés. Sa philosophie des images prend ainsi la forme d'une iconologie dont l'ambition est de rendre compte de tous les arts et de toutes les sciences qui ne procèdent nécessairement qu'à travers et par les images. Comme il l'écrit: " notre esprit n'agit que par images en la plupart de ses operations, \& il a sçu trouver des signes \& des figures sensibles pour nous exprimer sa pensee \& ses desseins les plus caches, d'une maniere ingenieuse ${ }^{25} »$. La liste de toutes les sciences qu'il présente mérite d'être citée in extenso, car on y perçoit comment chacune de ces sciences se trouve fondée sur un processus imaginatif :

«LA THÉOLOGIE fait les Images des choses surnaturelles, et divines, pour tascher de concevoir des veritez et des mysteres, qui sont d'eux mesmes incompréhensibles. LA PHILOSOPHIE a ses images dans ses actions, et c'est de leur diverse vûe que naissent toutes les disputes, et les contestations des Sçavans. Parce que comme ces Images se voient diversement selon les divers points dont elles sont regardées, ce qui change les situations, il arrive dans les ecoles ce qui arrive dans les Academies des Peintres, où ils copient le mesme modele, et font tous diverses figures, parce que l'un void ce modèle de front, un autre à demy de costé, un autre en tiers, un autre à dos. Il en est de mesme des choses qu'un esprit prevenu regarde, il les void de tout autre sens qu'un esprit qui n'est pas préoccupé. Et c'est cette perspective de la contemplation et de l'Estude, qui est la source infaillible de toutes les disputes des Sçavans sur une mesme matiere.

LA JURISPRUDENCE est l'image du bien public, que nous nous representons comme une sorte de corps, dont le Souverain est le chef, les Magistrats et la Noblesse les parties les plus considerables, et le peuple les autres membres [...]

L'HISTOIRE est la Peinture des événements, des desseins, des entreprises, et des mouvemens de ce corps [...]

LA MÉDECINE n'est qu'une image de la constitution intérierue, et extérieure du corps de l'homme et de ses affections, et de ses organisations pour ces fonctions vitales [...]

L'ASTRONOMIE a remply le ciel d'images pour en expliquer les figures et les mouvemens

L'ARITHMÉTIQUE peint les nombres pour soulager la memoire, et l'imagination

LA MUSIQUE a fait les yeux juges de tous les accords et de toutes les harmonies

LA GÉOMÉTRIE mesure toutes choses par lignes, par angles, et par Figures

Enfin tout LA MATHÉMATIQUE estant une science demonstrative ne consiste qu'en images

LA GRAMMAIRE est comme dit un de nos Poëtes 'un art ingénieux de peindre la parole, et parler aux yeux' [...]

LA FABLE ancienne estoit une Philosophie en Images

LA POÉSIE dont le propre est de feindre, est une faiseuse d'images

L'ÉLOQUENCE a ses figures, et la Rhetorique enseigne l'Art de Persuader par images $[\ldots]^{26} »$.

14 Comme on peut le constater, l'iconic turn n'a jamais été poussé aussi loin. Mais même dans cette vaste entreprise épistémologique qu'est la philosophie des images de Ménestrier, il apparaît quelque peu difficile de faire la part entre ce qui concerne strictement un savoir sur les images et un savoir par les images. C'est à vrai dire l'une des dernières tentatives de conciliation de ces deux voies qui apparaîtront par la suite comme des opposés : selon le bel anagramme de Ménestrier ${ }^{27}$, le caligo et la logica, c'està-dire le savoir mystérieux des anciens fondé sur l'épistémè de l'analogie, d'un côté, et la 
logique des modernes fondée sur l'épistémè de la représentation, de l'autre. Autrement dit, la rationalité de la philosophie aurait supplanté une pensée symbolique reléguée dans le domaine de l'irrationalité.

C'est en effet sur les ruines de cette improbable "philosophie des images "-deux termes qui vont finalement apparaître comme antagonistes - que va naître à la fin du XVIII ${ }^{\mathrm{e}}$ siècle l'idéologie, comprise comme science des idées remettant en cause le principe d'un savoir fondé sur l'image, savoir à l'origine de l'iconologie à la Ripa. Et c'est sur ce point qu'il est intéressant de revenir à l'argumentation de Mitchell. Il démontre en effet de manière assez convaincante que si l'idéologie s'impose comme nouvelle science des idées, répudiant les « idoles de l'esprit ", elle ne put s'empêcher de recourir à de nouvelles sortes d'images destinées à la prémunir contre toute mystification ou idolâtrie ${ }^{28}$. L'idéologie, qui apparait historiquement comme une science iconoclaste des idées ayant pour mission de mettre à bas les idoles, finit par prendre les traits d'une nouvelle forme d'idolâtrie, promouvant des formes abstraites et idéales comme les seules vraies et authentiques images qui soient. Bref, nous aurions affaire à une espèce d'ideolatrie, si l'on reprend la racine grecque eidos ou le mot latin idea, l'idée étant conçue comme vraie forme remplaçant l'eidolon qu'est le simulacre. En ce sens, l'idéologie selon son acception première (science des idées) est une idéologie selon son acception seconde qui est la nôtre aujourd'hui (système de valeurs et d'intérêts).

Mais la même chose peut être dite de l'iconologie : toute iconologie est une idéologie. Tout savoir sur les images reste un savoir par les images ou un savoir déterminé par des représentations. Telle est la dimension autoréflexive des Visual Studies, bien présente dans l'œuvre de Mitchell dont l'objectif pourrait être le suivant: étudier les représentations des représentations, l'imaginaire de l'imagination et l'imagination de l'imagerie, ce que résume assez bien sa formule «seing viewing " qui fait des Visual Studies une forme de méta-iconologie. Mais là encore, on peut procéder à une sorte d'anamnèse épistémologique. Revenons en effet, en guise de conclusion, à mon hypothèse principale ayant trait à la généalogie de l'idéologie comme iconologie et la généalogie de l'iconologie comme idéologie. J'ai cherché à démontrer que l'iconologie, en son sens originel, correspondait à ce que les images disent plutôt qu'à ce que nous disons à propos des images. Or il convient de regarder d'un peu plus près les théories $\mathrm{du}$ XVII siècle. On se rend alors compte que les iconologistes de cette époque étaient parfaitement conscients que toute pictura loquens, c'est-à-dire toute image parlante, est fondamentalement une image parlée. Ce qui était en jeu n'était donc pas tant ce que nous disons à propos des images ou ce que les images nous disent, que ce que nous disons à travers les images. Toute image n'était pas pensée comme parlant d'elle-même, mais comme animée par une voix off, que celle-ci prenne la forme d'un texte accompagnateur ou de la parole vive. Or une telle conception opère bien le lien entre les deux sens du mot iconologie, compris à la fois comme le logos des images et comme le logos sur les images, qui ne sont à vrai dire que les deux faces d'une même médaille. L'image provoque le langage et, en retour, le discours a le pouvoir d'animer littéralement l'image. En ce sens, l'iconologie est bien opposée à l'idolâtrie. Pour ne donner qu'un seul exemple illustrant cette opposition, voici ce qu'écrit un autre jésuite du XVII ${ }^{\mathrm{e}}$ siècle, Pierre Le Moyne, dans ses Peintures morales qui prennent la forme d'une galerie de tableaux littéraires: «Les Prestres du Paganisme s'enfermoient dans les Idoles de leurs Dieux, et parloient au Peuple par leur bouche, afin d'avoir plus 
d'authorite et se faire escouter religieusement et avec une sorte de culte ${ }^{29} »$. Ce passage pourrait illustrer parfaitement l'idolâtrie comme idéologie, expression d'un pouvoir opérant à travers les images. Mais en fait, il sert d'exemple à Le Moyne pour illustrer la prosopopée, figure du discours à travers laquelle un être absent ou imaginaire est représenté comme parlant. Dans la rhétorique de l'époque, on utilisait le mot grec enargeia pour désigner ce pouvoir d'animation. Rappelons que, pour Aristote, ce terme renvoie aux représentations mentales produisant l'effet de vie et de présence. Moins que le résultat, c'est le processus de représentation et les effets produits par cette représentation qui importent. Le but est de persuader et de transformer l'auditeurspectateur en l'impliquant dans la représentation. Car la fonction ultime de l'éloquence est bien le movere. Ce qui compte donc c'est l'interaction entre le sujet percevant et l'objet perçu. Or n'est-ce pas cette interaction qui est devenu l'objet principal des Visual Studies? Comme Mitchell y insiste lui-même, l'accent porté par l'analyse des cultures visuelles se déplace des choses vues vers le processus de la vision elle-même, mais une vision animée par le désir, et c'est en cela que les Visual Studies franchissent un pas supplémentaire en direction non plus de l'image pensante ou parlante, mais bien de l'image désirante, sujet et objet du désir.

\section{BIBLIOGRAPHIE}

Bacon Francis, Novum Organum [Londres, 1620], introduction, traduction et notes par Michel Malherbe et Jean-Marie Pousseur, Paris, PUF, 1986.

Baert Barbara, Lehmann Ann-Sophie, Akkerveken Jenke Van den, New Perspectives in Iconology, Visual Studies and Anthropology, Leuven (ed.), ASP, 2012.

Bryson Norman, Holly Michael Ann, Moxey Keith (ed.), Visual Theory. Painting and Interpretation, Cambridge, HarperCollins, 1991.

Caussin Nicolas, Electorum symbolorum et parabolarum historicarum syntagmata ex Horo, Clemente, Epiphanio et aliis, cum notis et observationibus, Paris, 1618.

Dekoninck Ralph, Ad imaginem. Statuts, fonctions et usages de l'image dans la littérature spirituelle jésuite du XVII siècle, Genève, Droz, 2005.

Dekoninck Ralph, « La Philosophie des images. D'une ontologie à une pragmatique de l'image », in Gérard Sabatier (dir.), Claude François Ménestrier, les jésuites et le monde des images, presses universitaires de Grenoble, Grenoble, 2009.

Dekoninck Ralph, « Entre logica et caligo. La Philosophie des images de Claude-François Ménestrier ", in Frédéric Cousinié et Clélia Nau (dir.), L'artiste et le philosophe. L'histoire de l'art à l'épreuve de la philosophie au XVII siècle, presses universitaires de Rennes, Rennes, 2011.

Dikovitskaya Margaret, Visual Culture. The Study of the Visual after the Cultural Turn, CambridgeLondon, The MIT Press, 2006. 
Gombrich Ernst Hans, «Icones symboliç. L'image visuelle dans la pensée néoplatonicienne », in Symboles de la Renaissance, Daniel Arasse et Georges Brunel (éd.), Paris, Presses de l'École normale supérieure, 1976.

Holly Michael Ann, Panofsky and the Foundations of Art History, Ithaca, Cornell University Press, 1984.

Latour Bruno, Petite réflexion sur le culte moderne des dieux faitiches, Paris, Synthélabo, 1996.

Latour Bruno, Weibel Peter (ed.), Iconoclash. Beyond the Image Wars in Science, Religion and Art, Cambridge, MIT Press, 2002.

Lavin Irving (ed.), Meaning in the Visual Arts: Views from the Outside. A Centennial Commemoration of Erwin Panofsky (1892-1968), Princeton, Princeton University Press, 1995.

Luther Martin, Luthers Werke. Kritische Gesamtausgabe, t. 28, Weimar, Hermann Bölhaus, 1903.

Ménestrier Claude-François, Les recherches du blason, Paris, Estienne Michallet, 1673.

Ménestrier Claude-François, La philosophie des images énigmatiques, Lyon, Hilaire Baritel, 1694.

Mitchell William J. Thomas, “What is Visual Culture?", in Irving Lavin (ed.), Princeton, Princeton University Press, 1995.

Mitchell William J. Thomas, Iconology: Image, Text, Ideology, Chicago and London, The University of Chicago Press, 1986.

Mitchell William J. Thomas, Iconologie: Texte, image, idéologie, Maxime Boidy et Stéphane Roth (trad.), Paris, Les Prairies ordinaires, 2009.

Mitchell William J. Thomas, What do pictures want? The Lives and Loves of Images, Chicago, The University of Chicago Press, 2005.

Moyne Pierre (Le), Les Peintures morales, où les passions sont representées par tableaux, par charactères, \& par questions nouvelles \& curieuses, Paris, Sébastien Cramoisy, 1640.

Ripa Cesare, Iconologia overo descrittione dell'imagini universali cavate dall'antichita et da altri luoghi, Rome, Per gli Heredi di Gio. Gigliotti, 1593.

\section{NOTES}

1. Notons qu'une telle situation diffère assez remarquablement de la tradition allemande où il ne semble y avoir aucune solution de continuité entre l'iconologie et la Bildwissenschaft.

2. M. Dikovitskaya, Visual Culture. The Study of the Visual after the Cultural Turn, Cambridge-London, The MIT Press, 2006.

3. M. A. Holly, Panofsky and the Foundations of Art History, Ithaca, Cornell University Press, 1984.

4. N. Bryson, M. A. Holly \& K. Moxey (ed.), Visual Theory. Painting and Interpretation, Cambridge, HarperCollins, 1991.

5. I. Lavin (ed.), Meaning in the Visual Arts: Views from the Outside. A Centennial Commemoration of Erwin Panofsky (1892-1968), Princeton, Princeton University Press, 1995.

6. W. J. T. Mitchell, "What is Visual Culture?", in I. Lavin (ed.), Meaning in the Visual Arts: Views from the Outside, op. cit., p. 207-215.

7. W. J. T. Mitchell, Iconology: Image, Text, Ideology, Chicago et London, The University of Chicago Press, 1986. Autre signe du temps, le nôtre cette fois, cet ouvrage inaugural vient seulement d'être traduit en français, soit donc plus de 20 ans après sa première parution, Iconologie: Texte, image, idéologie, M. Boidy et S. Roth (trad.), Paris, Les Prairies ordinaires, 2009. 
8. W. J. T. Mitchell, Iconology, op. cit., p. 2.

9. L'iconologie est ainsi définie comme «the study of the "logos" (the words, ideas, discourse, or "science") of "icons" (images, pictures, or likenesses). It is thus a "rhetoric of images" in a double sense: first, as a study of "what to say about images" - the tradition of "art writing" that goes back to Philostratus's Eikones, and is centrally concerned with the description and interpretation of visual art; and second of "what images say" - that is, the ways in which they seem to speak for themselves by persuading, telling stories, or describing ", Ibid., p. 1-2.

10. Ce que traduit assez bien le titre de l'une de ses dernières publications : W. J. T. Mitchell, What do pictures want? The Lives and Loves of Images, Chicago, The University of Chicago Press, 2005.

11. W. J. T. Mitchell, Iconology, op. cit., p. 2.

12. M. Luther, Luthers Werke. Kritische Gesamtausgabe, t. 28, Weimar, H. Bölhaus, 1903, p. 554.

13. «[...] les idoles et les notions fausses qui, s'étant déjà emparées de l'entendement humain, s'y sont fixées profondément, assiègent l'esprit au point que la vérité y trouve un accès difficile». F. Bacon, Novum Organum [Londres, 1620], introduction, M. Malherbe et J.-M. Pousseur (trad. et notes), Paris, PUF, 1986, p. 110.

14. «[...] human understanding, like a false mirror receiving rays irregularly, distorts and discolors the nature of things by mingling its own nature with it». W. J. T. Mitchell, Iconology, op. cit., p. 34 .

15. "The worship of graven images in the dark groves and caves of heathen superstition has given way to a superstitious belief in the power of graven mental images that reside in the dark cave of the skull ", ibid., p. 165, M. Boidy et S. Roth (trad.), Paris, Les Prairies ordinaires, 2009, p. 252.

16. W. J. T. Mitchell, Iconology, op. cit., p. 4.

17. «Insofar as these ideas are understood as images - as pictorial, graphic signs imprinted or projected on the medium of consciousness - then ideology, the science of ideas, is really an iconology, a theory of imagery ", Ibid., p. 164, traduction française p. 251.

18. N. Caussin, Electorum symbolorum et parabolarum historicarum syntagmata ex Horo, Clemente, Epiphanio et aliis, cum notis et observationibus, Paris, 1618.

19. Voir R. Dekoninck, Ad imaginem. Statuts, fonctions et usages de l'image dans la littérature spirituelle jésuite du XVII siècle, Genève, Droz, 2005, p. 38-40.

20. Une telle conception est encore bien présente à cette même époque dans les Icones symbolicce de Cristoforo Giarda (Milan, 1626), recueil d'« images symboliques » qui s'imposent comme autant de traces visibles des Idées situées dans l'esprit de Dieu. Elles ont donc une réelle valeur épiphanique, chaque objet visible donnant quelque aperçu de l'invisible dont il est le reflet dégradé. Voir E. H.Gombrich, «Icones symbolica. L'image visuelle dans la pensée néoplatonicienne ", in Symboles de la Renaissance, D. Arasse et G. Brunel (éd.), Paris, Presses de l'École normale supérieure, 1976, p. 17-29.

21. N. Caussin, Electorum symbolorum, op. cit., 7 ro L'Iconologia de Cesare Ripa (Rome, 1593) avait déjà ouvert la voie à une telle "rationalisation» du symbole en proposant un dictionnaire alphabétique des représentations des vices et des vertus destinées à servir de modèles aux artistes dans l'inventio de leurs compositions. Ces allégories aux attributs bien codifiés transforment les imagines agentes des anciens arts de mémoire (images qui d'aide-mémoire étaient devenues, à la Renaissance, l'incarnation des idées) en figures du discours peint.

22. C. Ripa, Iconologia overo descrittione dell'imagini universali cavate dall'antichita et da altri luoghi, Rome, Per gli Heredi di Gio. Gigliotti, 1593, proemio.

23. «El primo modo furono trattate da molti antichi, fingendo le imagini delle Deità, le quali non sono altro, che veli, ò vestimenti da tenere ricoperta quella parte dei Filosofia, che riguarda la generatione, \& la corrottione delle cose naturali, ò la dispositione de' Cieli, ò l'influenza delle Stelle, ò la fermezza della Terra, ò alter simili cose, le quali con un lungo studio ritrovarono per avanzare in questa cognizione la plebe, e accioché non egualmente i dotti e gl'ignoranti potessero 
intendere e penetrare le cagioni delle cose, se le andavano copertamente communicando fra se stessi e copertamente ancora per mezzo di queste imagini le lasciavano a' posteri, che dovevano agli altri essere superiori di dignità e sapienza », ibid.

24. Je me permets de renvoyer à R. Dekoninck, «La Philosophie des images. D'une ontologie à une pragmatique de l'image ", in G. Sabatier (dir.), Claude François Ménestrier, les jésuites et le monde des images, presses universitaires de Grenoble, Grenoble, 2009, p. 103-113 ainsi qu'à R. Dekoninck, « Entre logica et caligo. La Philosophie des images de Claude-François Ménestrier », in Fr. Cousinié et Cl. Nau (dir.), L'artiste et le philosophe. L'histoire de l'art à l'épreuve de la philosophie au XVII siècle, presses universitaires de Rennes, Rennes, 2011, p. 199-211.

25. Cl.-Fr. Ménestrier, Les recherches du blason, Paris, Estienne Michallet, 1673, avertissement.

26. Ibid.

27. «Aulugelle n'est pas le seul qui ait pû donner le nom de nuit à ses ouvrages: toutes nos sciences devroient porter le mesme nom. Caligo n'est pas seulement l'Anagramme du nom de la Logique, il est la définition mesme de nostre Philosophie bien mieux que celle qu'on lui attribuë de connoissance des choses Divines et humaines, dans lesquelles on peut dire qu'elle est aveugle ». Cl.-Fr. Ménestrier, La philosophie des images énigmatiques, Lyon, Hilaire Baritel, 1694, préface.

28. " [...] ideology, then, which begins historically as an iconoclastic "science of ideas" designed to overturn "idols of the mind", winds up being characterized as itself a new form of idolatry, an ideolatry or eidolatry promoting abstract and ideal forms as true images. It will finally lead to the triumph of symbol over allegory ». W. J. T. Mitchell, Iconology, op. cit., p. 34. Voir également B. Latour, Petite réflexion sur le culte moderne des dieux faitiches, Paris, Synthélabo, 1996, ainsi que B. Latour et P. Weibel (ed.), Iconoclash. Beyond the Image Wars in Science, Religion and Art, Cambridge, MIT Press, 2002.

29. P. Le Moyne, Les Peintures morales, où les passions sont representées par tableaux, par charactères, \& par questions nouvelles \& curieuses, Paris, Sébastien Cramoisy, 1640, avertissement, n.p.

\section{RÉSUMÉS}

Loin de se réduire à la question des rapports entre texte et image et du logocentrisme, l'étude inédite des rapports entre Visual Studies et iconologie montre que tout logos des et sur les images engage une dimension théorique mais aussi pratique, idéologique voire idolâtrique. S'appuyant sur une généalogie de l'iconologie, de Philostrate à Cesare Ripa, de Luther à Bacon, des jésuites à W. J. T. Mitchell, une archéologie des Visual Studies montre que, successivement savoir par l'image et savoir sur l'image, l'iconologie dépasse d'emblée la sphère artistique pour situer la question du sens et du pouvoir des images dans le champ rhétorique, religieux et politique.

\section{INDEX}

Mots-clés : iconologie, idéologie, idolâtrie, image, rhétorique, Visual Studies

Personnes citées : Mitchell (William J. Thomas) 


\section{AUTEUR}

\section{RALPH DEKONINCK}

Ralph Dekoninck est professeur d'histoire de l'art des temps modernes à l'université catholique de Louvain où il codirige le Centre d'analyse culturelle de la première modernité (GEMCA). Spécialiste de l'histoire des pratiques et des théories de l'image dans le christianisme, ses travaux actuels portent sur les rapports entre théologie de l'image et théorie de l'art durant la première modernité ainsi que sur les figures de l'idolâtrie dans l'imaginaire moderne. Parmi ses publications, personnelles et collectives, on peut citer : Ad Imaginem. Statuts, fonctions et usages de l'image dans la littérature spirituelle jésuite du XVII siècle, Genève, Librairie Droz, 2005 ; Fou comme une image. Puissance et impuissance de nos idoles, Bruxelles, Éditions Labor, 2006 ; avec A. Guiderdoni et N. Kremer, Aux limites de l'imitation. L'ut pictura poesis à l'épreuve de la matière, Amsterdam, Éditions Rodopi, 2009 ; avec D. Lories, L'art en valeurs, Paris, L'Harmattan, 2011. 